Classification

Physics Abstracts

$61.50 \mathrm{C}-64.70$

\title{
The Mullins-Sekerka instability in directional solidification of quasi-azeotropes
}

\author{
C. Misbah \\ Groupe de Physique des Solides de l'Ecole Normale Supérieure, associé au Centre National \\ de la Recherche Scientifique, Université Paris VII, 2, place Jussieu, 75251 Paris Cedex 05, France
}

(Reçu le 3 décembre 1985, accepté le 21 février 1986)

\begin{abstract}
Résumé. - Nous étudions la stabilité du front plan lors de la solidification directionnelle d'un quasi-azéotrope. Nous montrons que la forme du diagramme de phase donne lieu à une forte réduction d'échelle de la courbe de bifurcation qui rend possible son exploration complète dans les expériences de solidification directionnelle usuelles. Nous montrons de plus, que, comme les azéotropes ne sont pas des mélanges dilués, il apparaît un « terme capillaire chimique » dans l'équation reliant les concentrations à l'interface. Nous estimons l'importance de cet effet : il est très faible dans les azéotropes métalliques mais, devrait être important dans le mélange ${ }^{3} \mathrm{He}-{ }^{4} \mathrm{He}$ et pourrait donner lieu à un changement qualitatif de la forme de la courbe de bifurcation. A l'aide d'un développement au troisième ordre en amplitude, nous étudions la nature (normale ou inversée) de la bifurcation. Nous montrons que, pour les alliages métalliques, et grâce à la réduction d'échelle du diagramme d'instabilité, le régime de bifurcation normale - qui n'est pas facilement explorable avec les mélanges dilués - devrait devenir accessible si on utilise des mélanges quasi-azéotropes.
\end{abstract}

\begin{abstract}
We investigate the stability of the planar front during directional solidification of a quasi-azeotrope. We show that the corresponding shape of the phase diagram induces a strong reduction of the scale of the bifurcation curve which makes its complete exploration feasible in standard directional solidification experiments. We show that the finite concentration of azeotropic mixtures results in a « chemical capillary term » in the equation relating interface concentrations. This effect, which turns out to be negligibly small for metallic azeotropes, should be significant for the ${ }^{3} \mathrm{He}-{ }^{4} \mathrm{He}$ azeotrope where it might give rise to a qualitative change of the bifurcation curve. We study the nature (normal or inverted) of the bifurcation with the help of a third order amplitude expansion. We show that, for metallic alloys, thanks to the reduction of scale of the instability diagram, the normal bifurcation regime - which cannot be easily reached with dilute mixtures - becomes accessible to standard experiments.
\end{abstract}

\section{Introduction.}

When dilute binary mixtures are submitted to directional solidification (i.e. the sample is pulled at constant velocity $\tilde{V}$ in an external thermal gradient (see Fig. 1)), the liquid-solid interface undergoes, when $\tilde{V}$ exceeds a critical value $\tilde{V}_{c}$, a transition from the planar configuration to a periodic cellular one. This morphological instability results from the competition between the destabilizing effect of solute diffusion and the stabilization due to the external thermal gradient and to capillary forces.

In directional solidification experiments, the three external control parameters are the drawing velocity of the sample $\tilde{V}$, the applied thermal gradient $\bar{G}$ and the concentration $\widetilde{C}_{\infty}$ of the liquid far from the solidification front.
For a given $\tilde{C}_{\infty}$, the shape of the bifurcation curve, first analysed by Mullins and Sekerka [1] (see also Wollkind and Segel [2]), is drawn in the $(\widetilde{G}, \widetilde{V})$ plane in figure 2 . The maximum critical velocity, usually called the "absolute stability limit ", $\tilde{V}_{\max }$ and the thermal gradient $\widetilde{G}_{\max }$ are respectively given by [3] :

$$
\begin{aligned}
& \tilde{V}_{\max }=a \tilde{C}_{\infty} \\
& \tilde{G}_{\max }=b \tilde{C}_{\infty}^{2}
\end{aligned}
$$

$a$ and $b$ are functions of the thermodynamic properties and the transport coefficients of the alloy. A rough estimate for typical materials [3] shows that $a$ and $b$ are respectively in the 10 and $10^{5}$ ranges, with $\tilde{V}$ in $\mathrm{cm} / \mathrm{s}, \tilde{G}$ in $\mathrm{K} / \mathrm{cm}$ and $\widetilde{C}_{\infty}$ in atomic per cent. 


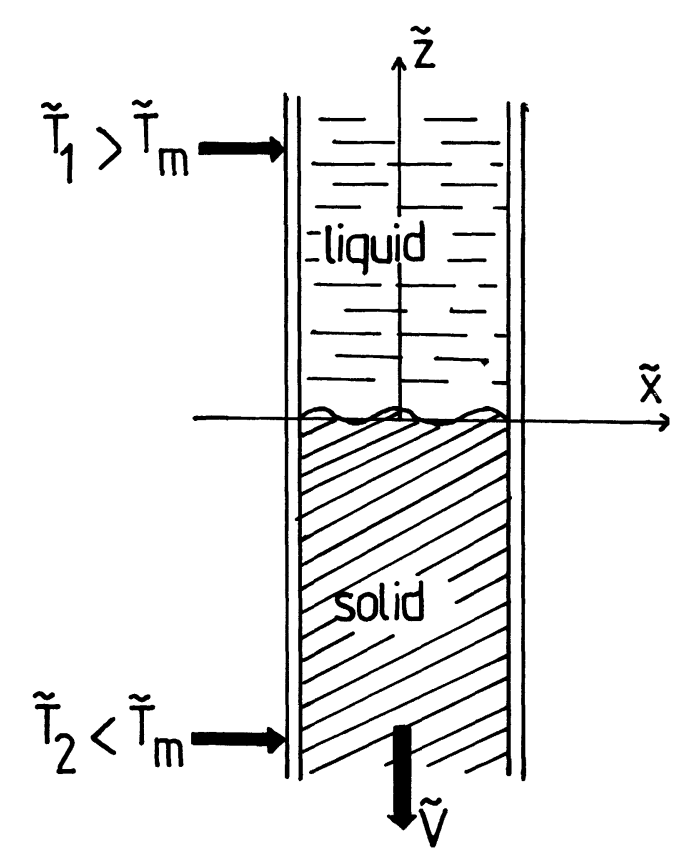

Fig. 1. - Directional solidification set up. $\tilde{T}_{m}$ is the melting temperature of the alloy.

In standard solidification experiments $\left({ }^{1}\right)$, the velocity of the sample may reasonably reach $10^{-1} \mathrm{~cm} / \mathrm{s}$. Lowering $\widetilde{V}_{\max }$ down to this order of magnitude would take extremely dilute mixtures with $\widetilde{C}_{\infty}$ of order at most $10^{-2} \%$. So, directional solidification experiments have only explored the small $\tilde{V}$, small $\tilde{G}$ part of the instability curve (see Fig. 2).

Furthermore, recent experiments [6] performed on impure $\mathrm{CBr}_{4}$ have shown that the bifurcation is subcritical (i.e. exhibits a finite hysteresis). This means that, when the pulling velocity is increased, the front deformation jumps from zero to a finite amplitude at the instability threshold (as does the order parameter at a first order phase transition). This result agrees with the prediction of Wollkind and Segel [2] and Caroli et al. [3]; it entails that non linear perturbation expansions, which result in the amplitude equation, cannot be used to calculate cellular front shapes close to threshold, since such expansions are not valid at finite deformation amplitudes.

As shown by Wollkind and Segel [2], for dilute mixtures, it is only at large velocities beyond the reach of standard experiments that the bifurcation can be expected to become normal (i.e. with a front deforma-

(1) In rapid solidification experiments, which use a pulsed laser [4] or electron beam [5] to melt a spot in the surface region, the resolidification velocity, which is directly related to that of the beam, can reach values larger than $\tilde{V}_{\max }$. In particular, and as predicted by Mullins and Sekerka [1], a "restabilization " of the planar front has been observed for such large velocities. However, the thermal profile is not well controlled in these experiments, which are therefore not well suited (at least up to now) to give a full quantitative characterization of the bifurcation.

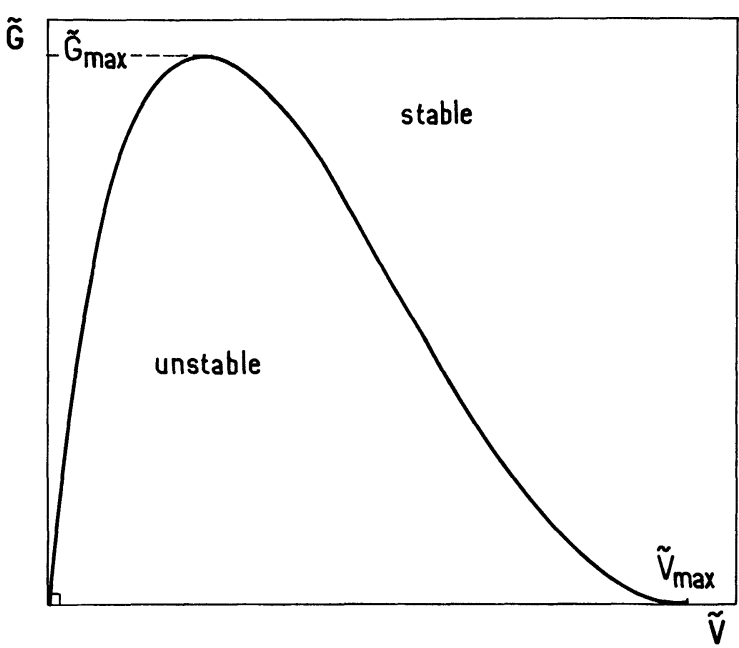

Fig. 2. - Schematic plot of the bifurcation curve in the $(\tilde{G}, \tilde{V})$ plane. $\tilde{G}$ is the physical temperature gradient and $\tilde{V}$ the velocity of the sample. The square near the-origin represents the accessible region in standard directional solidification experiments.

tion amplitude increasing continuously from zero above $\tilde{V}_{\mathrm{c}}$ ).

So, it seems quite desirable to find physical systems which would allow experimental access to the normal bifurcation regime. Indeed, this would permit to compare cellular front shapes close above threshold in the normal and subcritical situations, and thereby, to settle important open questions, in particular :

- are the cusp singularities of cellular fronts close above threshold observed up to now a general feature, or are they due to the subcriticality of the bifurcation ?

- is the wavelength selection above threshold strongly modified by a change in the nature of the bifurcation?

Experimental answers to such questions are, in our opinion, of major importance to guide further progress in the theoretical analysis.

In this article, we show that quasi-azeotropic binary mixtures are good candidates to the experimental observation of normal Mullins-Sekerka bifurcations.

The corresponding equilibrium phase diagram is sketched in figure 3 . It immediately appears that the main difference, for our purpose, between this and the dilute case lies in the quadratic dependence of the equilibrium interface temperature on concentration (as measured from the azeotropic one, $\widetilde{C}_{0}$ ).

We show, from the linear stability analysis of the planar front, that this feature results in a strong reduction of the scale of the bifurcation curve. Namely, for departures from $\tilde{C}_{0}$ in the atomic \% range, the absolute stability limit $V_{\max }$ and the maximum thermal gradient $G_{\max }$ are reduced (as compared with dilute mixtures with $\tilde{C}_{\infty} \sim 1 \%$ ), by factors of order, respectively, $10^{2}$ and $10^{4}$. This should make the complete exploration of the bifurcation curve experimentally feasible. 


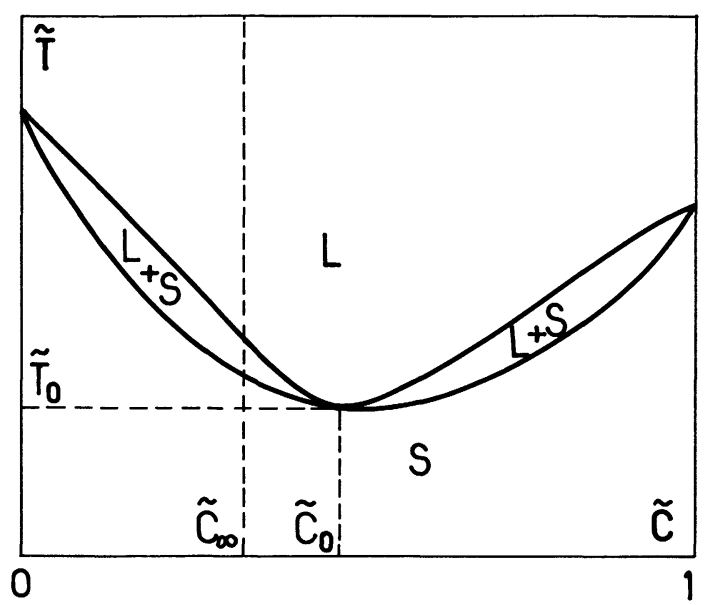

Fig. 3. - Schematic plot of the phase diagram of the alloy in the $(\tilde{T}, \widetilde{C})$ plane. $\left(\tilde{T}_{0}, \tilde{C}_{0}\right)$ is the azeotropic point, $\tilde{C}_{\infty}$ is the (imposed) concentration in the liquid phase far from the solidification front.

Besides the phase diagram shape effect, there is another difference between the azeotropic and dilute cases : for mixtures of finite concentration, there appears, in the equation relating the concentrations of the two phases at the interface, a contribution proportional to the interface curvature, that we call from now on " chemical capillary term ». This term, which results from the conditions of local thermodynamic equilibrium of the two species at the interface $\left(^{2}\right)$, is strictly zero only when the solute concentration in the reference state vanishes.

We show that the presence of a non-negligible capillary term may give rise to a qualitative change in the shape of the Mullins-Sekerka bifurcation curve. Indeed, at zero "chemical capillarity", changing $\tilde{C}_{\infty}-\tilde{C}_{0}$ into its opposite leaves this curve invariant. When the magnitude of the chemical capillary term increases, there appears an asymmetry between the "hyper" and the "hypoazeotrope" with equal $\left|\tilde{C}_{\infty}-\tilde{C}_{0}\right|$; namely, in the high velocity part of the bifurcation curve, the planar front state is stabilized or destabilized, depending on the sign of $\left(\tilde{C}_{\infty}-\tilde{C}_{0}\right)$. This asymmetry becomes maximum at a critical value of the chemical capillarity, beyond which the destabilized bifurcation curve no longer has a limit of absolute stability.

From our semi-quantitative estimates of the magnitude of the chemical capillary parameter, it appears that this effect should be practically negligible for most azeotropes. However, it will probably be significant for ${ }^{3} \mathrm{He}-{ }^{4} \mathrm{He}$ quasi-azeotropic mixtures, on which experiments can be expected to be carried out in the near future.

${ }^{2}$ ) We only consider materials with atomically rough liquid-solid interfaces, for which interface kinetics can safely be taken as instantaneous (as compared with the diffusive time scale).
In order to study the nature of the Mullins-Sekerka bifurcation, following Wollkind and Segel [2], we perform a non-linear analysis up to third order in the front deformation amplitude. From this, we get the analytic expression of the Landau constant $a_{1}$ (the coefficient of the cubic term in the amplitude expansion), the sign of which determines whether the bifurcation is normal or subcritical. We have calculated $a_{1}$ numerically in the limit, appropriate for metallic azeotropes, of zero chemical capillarity. We find that, in spite of the quadratic concentration dependence of the quasi-azeotrope interface equilibrium temperature, $a_{1}$ exhibits a behaviour qualitatively similar to that for dilute systems. Namely, the bifurcation becomes normal above a velocity of order, typically, $\tilde{V}_{\max } / 10$.

This means that, thanks to the above-mentioned scale reduction of the bifurcation curve, the normal bifurcation regime should become accessible to standard directional solidification experiments.

The scheme of this paper is as follows. In section 2, we write down the basic equations of the problem and compare them briefly to those for dilute mixtures. In section 3, we study the linear stability of the planar front and derive the bifurcation equations. Then, we discuss in some detail the order of magnitude of the chemical capillary term for metallic azeotropes and for helium mixtures, and the shapes of the corresponding bifurcation curves. In section 4 , we perform the third order amplitude expansion and present the numerical results relative to metallic azeotropes. Our results and conclusions are summed up in section 5 .

\section{Basic equations.}

We consider the following situation (Fig. 1) : a binary mixture $A_{C} B_{1-C}(\tilde{C}$ is the mass fraction of component

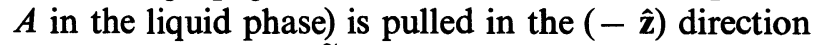
at constant velocity $\tilde{V}$. The concentration far ahead of the solidification front is fixed at a value $\tilde{C}_{\infty}$ close to the azeotropic composition $\tilde{C}_{0}$ (Fig. 3). The system is quasi-infinite (on the scale of all wavelengths of interest) in the $(\tilde{x}, \tilde{y})$ directions. The temperatures in the liquid and the solid are denoted by $\widetilde{T}_{\mathrm{L}}$ and $\widetilde{T}_{\mathrm{S}}$ respectively, the concentration in the solid by $\tilde{C}_{\mathrm{s}}$. All the extensive thermodynamic quantities are defined per unit mass.

As usual, we neglect mass diffusion in the solid, since the corresponding diffusion coefficient $D_{\mathrm{S}} \sim 10^{-11}$. $10^{-11} \mathrm{~cm}^{2} / \mathrm{s}$, while in the liquid $D_{\mathrm{L}} \sim 10^{-5} \mathrm{~cm}^{2} / \mathrm{s}$.

The temperature fields in both phases are assumed to be in a quasi-steady state and satisfy Laplace's equation. This assumption is justified by the fact that thermal diffusivities are several orders of magnitude larger than chemical diffusivities, so that the temperature fields relax very quickly to their steady state values $\left({ }^{2}\right)$. One must however point out that this assumption remains valid only if the wavelengths of the "most dangerous" modes are small compared with the thermal diffusion length $\left(D_{\mathrm{L}, \mathrm{S}}^{\text {th }} / \not{D}\right), D_{\mathrm{L}, \mathrm{S}}^{\text {th }}$ being the heat diffusivity in the liquid or the solid phase. 
We shall see later that this is satisfied except for pulling velocities very close to the absolute stability limit (if any).

Following Wollkind and Segel [2] (WS), we only study the stability against the simplest type of interfacial deformations, namely 1-dimensional ones.

From now on, we use the following dimensionless variables :

$$
\begin{aligned}
(x, z) & =(\tilde{x}, \tilde{z}) /\left(\frac{D_{\mathrm{L}}}{\tilde{V}}\right), & t & =\tilde{t} /\left(\frac{D_{\mathrm{L}}}{\tilde{V}^{2}}\right) \\
T & =\frac{\tilde{T}}{\tilde{T}_{0}}, & u & =\frac{\tilde{C}-\tilde{C}_{0}}{\tilde{C}_{\infty}-\tilde{C}_{0}} K \equiv \frac{\tilde{C}-\tilde{C}_{0}}{\tilde{u}_{\infty}} K
\end{aligned}
$$

where the tilted variables denote the physical ones. $D_{\mathrm{L}}$ is the concentration diffusion coefficient in the liquid, $\tilde{T}_{0}$ the azeotropic temperature and $K$ the equilibrium concentration distribution coefficient; $K=\sqrt{\left(\alpha_{\mathrm{L}} / \alpha_{\mathrm{S}}\right)}$, where $\alpha_{\mathrm{L}}$ and $\alpha_{\mathrm{S}}$ are the curvatures of the liquidus and solidus at $\tilde{C}=\tilde{C}_{0}$ and $\tilde{T}=\widetilde{T}_{0}$ : $\alpha_{\mathrm{L}, \mathrm{S}}=\left.\frac{1}{2} \frac{\mathrm{d}^{2} \tilde{T}_{\mathrm{L}, \mathrm{s}}}{\mathrm{d} \tilde{C}_{\mathrm{L}, \mathrm{s}}^{2}}\right|_{\tilde{c}=\tilde{c}_{0}}$

Let $z=\varepsilon \xi(x, t)$ be the (reduced) position of the interface in the laboratory frame, $\varepsilon$ being a smallness parameter. The equations governing mass and heat transport are then :

$$
\begin{array}{ll}
\begin{array}{l}
\left(\nabla^{2}+\frac{\partial}{\partial z}-\frac{\partial}{\partial t}\right) u_{\mathrm{L}}=0 \\
\nabla^{2} T_{\mathrm{L}}=0
\end{array} & \begin{array}{l}
z>\varepsilon \xi(x, t) \\
\nabla^{2} T_{\mathrm{S}}=0
\end{array} \\
z<\varepsilon \xi(x, t) .
\end{array}
$$

On the interface (for $z=\varepsilon \xi(x, t)$ ), temperature continuity and mass $\left({ }^{3}\right)$ and heat balance take the form :

$$
\begin{gathered}
T_{\mathrm{L}}=T_{\mathrm{S}} \\
\mathbf{n} \cdot\left(n \nabla T_{\mathrm{S}}-\nabla T_{\mathrm{L}}\right)=0 \\
\mathbf{n} \cdot \nabla u_{\mathrm{L}}=\left(u_{\mathrm{S}}-u_{\mathrm{L}}\right) \mathbf{V}_{\mathrm{I}} \cdot \mathbf{n}
\end{gathered}
$$

where $\mathbf{n}$ is the unit normal to the interface pointing into the liquid, $V_{I}$ is the velocity of the interface and $n \equiv K_{\mathrm{S}} / K_{\mathrm{L}} ; K_{\mathrm{s}}$ and $K_{\mathrm{L}}$ are the heat diffusion coefficients in the solid and liquid respectively.

These interface conditions must be supplemented with kinetic equations relating the mass currents of the two species to the chemical potential differences across the interface.

We assume that the interface is microscopically rough, and therefore that the kinetic is fast enough for local chemical equilibrium to be realized at the solid liquid interface. That is :

$$
\mu_{\mathrm{L}}^{i}=\mu_{\mathrm{s}}^{i} \quad(i=A, B) .
$$

$\left(^{3}\right)$ We neglect, for simplicity, all hydrodynamic effects [7] This implies, in particular, that we neglect the density difference between the two phases.
Then, developing the chemical potentials $\mu_{\mathrm{L}}^{i}$ and $\mu_{\mathrm{S}}^{i}$ around the reference azeotropic point $\hat{T}=\widetilde{T}_{0}, \tilde{C}=\tilde{C}_{0}$, and $\tilde{p}=\tilde{p}_{0}\left(\tilde{p}_{0}\right.$ is the azeotropic pressure), we show in appendix $A$ that equations (5) can be rewritten :

$$
\begin{aligned}
& u_{\mathrm{S}}=K u_{\mathrm{L}}-d_{0}^{\mathrm{c}} \kappa \\
& T_{\mathrm{L}}=1+M u_{\mathrm{L}}^{2}-d_{0} \kappa
\end{aligned}
$$

where

$$
\begin{aligned}
& d_{0}^{\mathrm{c}} \equiv \frac{K \gamma \tilde{V}\left(1-\tilde{C}_{0}\right) \kappa}{D_{\mathrm{L}} u_{\infty}\left(\frac{\partial \mu_{\mathrm{S}}^{\mathrm{A}}}{\partial C}\right) \rho_{\mathrm{S}} \mathcal{L}}\left[\tilde{T}_{0}\left(\Delta S^{\mathrm{B}}-\Delta S^{\mathrm{A}}\right)-\frac{\mathcal{L}}{\rho_{\mathrm{S}}} \frac{\partial \rho_{\mathrm{S}}}{\partial C_{\mathrm{S}}}\right] \\
& d_{0} \equiv \frac{\gamma \tilde{V}}{D_{\mathrm{L}} \rho_{\mathrm{S}} \mathcal{L}} ; \quad M=\frac{\alpha_{\mathrm{L}} \tilde{u}_{\infty}^{2}}{\tilde{T}_{0} K^{2}}
\end{aligned}
$$

$\rho_{\mathrm{S}}$ is the solid density, $\mathcal{L}$ the latent heat of fusion per unit mass of the azeotrope, $\kappa$ the surface curvature defined as positive for a convex solid. $\Delta S^{i} \equiv S_{\mathrm{L}}^{i}-S_{\mathrm{S}}^{i}$ where $S_{\mathrm{L}}^{i}$ and $S_{\mathrm{S}}^{i}$ are the partial entropies of species $i$ in the liquid and solid phases respectively and are related to the specific entropies $S_{\mathrm{L}, \mathrm{S}}$ of the mixture by Euler's theorem :

$$
\begin{aligned}
& S_{\mathrm{L}, \mathrm{S}}^{\mathrm{A}}=S_{\mathrm{L}, \mathrm{S}}+\left(1-\tilde{C}_{0}\right) \frac{\partial S_{\mathrm{L}, \mathrm{S}}}{\partial \tilde{C}_{\mathrm{L}, \mathrm{S}}} \\
& S_{\mathrm{L}, \mathbf{S}}^{\mathrm{B}}=S_{\mathrm{L}, \mathbf{S}}-\tilde{C}_{0} \frac{\partial S_{\mathrm{L}, \mathbf{S}}}{\partial \tilde{C}_{\mathrm{L}, \mathrm{S}}} .
\end{aligned}
$$

Let us point out that equation (6a) contains a capillary term which is absent in the case of dilute binary mixture. Indeed, in that case, the natural reference state has zero concentration and $\left(\partial \mu_{\mathbf{s}}^{\mathbf{A}} / \partial \widetilde{C}\right) \rightarrow \infty$ for $\tilde{C}_{0} \rightarrow 0$, while the term in square brackets in equation (7) remains finite.

The order of magnitude of this term for different types of materials will be discussed at the end of this section. Let us simply mention at this stage that $d_{0}^{\mathrm{c}}$ may, in principle, be either positive or negative.

Note also, that the concentration-dependent term in the Gibbs-Thomson equation (6b) is quadratic. This is simply due to the fact that the reference state corresponds to an extremum of the coexistence curves in the $(\widetilde{T}, \widetilde{C})$ plane.

We finally impose the following boundary conditions :

(i) the temperature is fixed at the two thermal contacts, namely :

$$
\begin{array}{lll}
\tilde{T}_{\mathrm{L}}=\tilde{T}_{1} & \text { at } & \tilde{z}=\tilde{L}_{1} \\
\tilde{T}_{\mathrm{S}}=\tilde{T}_{2} & \text { at } & \tilde{z}=-\tilde{L}_{2} .
\end{array}
$$

The dimensions $\tilde{L}_{1,2}$ of "the thermal box" are assumed to be much smaller than the thermal diffusion length [3];

(ii) the concentration is fixed at a value $\widetilde{C}_{\infty}$ far ahead of the front in the liquid phase - i.e., at a distance much larger than $D_{\mathrm{L}} / \tilde{V}$. So, for all practical purposes, 
this amounts to :

$$
\tilde{C}_{\mathbf{L}}(\tilde{z} \rightarrow \infty)=\tilde{C}_{\infty} .
$$

The set of equations (3)-(10) completely describes the motion of the solidification front. These equations admit a simple solution, namely the steady state planar interface one. It is given by :

$$
\begin{aligned}
& \left.\begin{array}{l}
T_{\mathrm{L}}^{0}=1+M+G z \\
u_{\mathrm{L}}^{0}=1+(K-1)\left(1-\mathrm{e}^{-z}\right)
\end{array}\right\} z>0 \\
& T_{\mathrm{S}}^{0} \rightleftharpoons 1+M+\frac{G}{n} z \quad z<0
\end{aligned}
$$

$G$, the reduced temperature gradient in the liquid phase is given by :

$$
G=\frac{T_{1}-n T_{2}+(1+M)(n-1)}{L_{1}+L_{2}} .
$$

\section{Linear stability analysis.}

We study the regression of fluctuations by considering solutions of form :

$$
\mathbf{v}=\mathbf{v}_{0}+\varepsilon \cos (q x) \mathrm{e}^{\sigma t} \mathbf{v}_{1}(z)
$$

where $v=\left(T_{\mathrm{L}}, u_{\mathrm{L}}, T_{\mathrm{S}}\right)$ and $\mathrm{v}_{0}$ is the planar interface solution given by equations (11).

The interface position is given by :

$$
z=\varepsilon \xi_{1}(x, t)=\varepsilon \cos (q x) \mathrm{e}^{\sigma t} .
$$

Substituting equation (13) into equations (3), one gets to first order in $\varepsilon$ :

$$
\begin{aligned}
& \left(\frac{\mathrm{d}^{2}}{\mathrm{~d} z^{2}}+\frac{\mathrm{d}}{\mathrm{d} z}-q^{2}-\sigma\right) u_{\mathrm{L}}^{1}(z)=0 \\
& \left(\frac{\mathrm{d}^{2}}{\mathrm{~d} z^{2}}-q^{2}\right) T_{\mathrm{L}}^{1}(z)=0 \\
& \left(\frac{\mathrm{d}^{2}}{\mathrm{~d} z}-q^{2}\right) T_{\mathrm{S}}^{1}(z)=0
\end{aligned}
$$

with

$$
\begin{gathered}
u_{\mathrm{L}}^{1}(\infty)=0 \\
T_{\mathrm{L}}^{1}\left(L_{1}\right)=T_{\mathrm{S}}^{1}\left(-L_{2}\right)=0 .
\end{gathered}
$$

The solutions of these equations can be written as :

$$
\begin{aligned}
& u_{\mathrm{L}}^{1}(z)=\beta_{1} \mathrm{e}^{-m z} \\
& T_{\mathrm{L}}^{1}(z)=\alpha_{1} \mathrm{e}^{-q z} \\
& T_{\mathbf{S}}^{1}(z)=\alpha_{1}^{\prime} \mathrm{e}^{q z}
\end{aligned}
$$

where

$$
m=\frac{1}{2}+\sqrt{\frac{1}{4}+q^{2}+\sigma} ; \text { with } \operatorname{Re}(m)>0 .
$$

In expressions (17b) and (17c), we have neglected the reflection of temperature fluctuations at $|z|=L_{1,2}$, since, as mentioned above, we assume that the length of the sample is much larger than the wavelengths of interest : $q L_{1,2} \gg 1$.

Substituting equations (13)-(14) in the interface boundary conditions (4) and (6) and expanding these resulting equations about $z=0$ to first order in $\varepsilon$, one obtains :

$$
\begin{gathered}
\alpha_{1}^{\prime}-\alpha_{1}+\frac{1-n}{n} G=0 \\
\alpha_{1}+n \alpha_{1}^{\prime}=0 \\
(1-m-K) \beta_{1}+(1-K)(K+\sigma)+d_{0}^{\mathrm{c}} q^{2}=0 \\
\alpha_{1}-2 M \beta_{1}+G-2 M(K-1)+d_{0} q^{2}=0 .
\end{gathered}
$$

The conditions of compatibility for system (18) gives the dispersion equation relating $\sigma$ and $q$ :

$$
(m+K-1)\left(\mathscr{C}-\beta q^{2}\right)=\sigma+K+K \beta \eta q^{2}
$$

where :

$$
\begin{aligned}
& \mathcal{C}=1-\frac{G}{M(K-1)(n+1)} \\
& \beta \equiv \frac{d_{0}}{2 M(K-1)} ; \quad \eta \equiv-\frac{2 d_{0}^{\mathrm{c}} M}{d_{0} K} .
\end{aligned}
$$

Note that, compared with the dilute case [2], the dispersion equation (19) contains an additional contribution which stems from the capillary term of equation (6a). In addition, the dimensionless parameters $\mathcal{C}$ and $\beta$ involve a factor $M$ proportional to $\tilde{u}_{\infty}^{2}$ (see Eq. (7)), while the corresponding factor for dilute mixtures [2] is proportional to $\tilde{u}_{\infty}$. As we shall see below, this difference is the source of the scale reduction of the bifurcation diagram.

The linear stability analysis completely follows that of WS. The stability of the planar front is determined by the sign of $\operatorname{Re} \sigma$. Since we look for the instability threshold, we are only interested in studying the condition of existence of neutral modes, defined by $\operatorname{Re} \sigma=0$.

We will first prove that equation (19) entails that, if $\operatorname{Re} \sigma=0$, then $\operatorname{Im} \sigma=0$. For this purpose, let us set :

$$
\sigma=\operatorname{Re} \sigma+i \operatorname{Im} \sigma=\sigma_{\mathrm{r}}+i \sigma_{\mathrm{I}} .
$$

Substituting equation (21) into equation (19) and assuming $\sigma_{\mathrm{r}}=0$, we find :

$$
\begin{aligned}
& K+K \beta \eta q^{2}-\frac{\mathcal{b}-\beta q^{2}}{\sqrt{2}}\left\{\sqrt{2}\left(K-\frac{1}{2}\right)+\right. \\
& \left.+\left[q^{2}+\frac{1}{4}+\sqrt{\left(q^{2}+\frac{1}{4}\right)^{2}+\sigma_{\mathrm{I}}^{2}}\right]^{1 / 2}\right\}=0 \\
& \sigma_{\mathrm{I}}\left\{1-\frac{\varepsilon-\beta q^{2}}{\sqrt{2}\left[q^{2}+\frac{1}{4}+\sqrt{\left(q^{2}+\frac{1}{4}\right)^{2}+\sigma_{\mathrm{I}}^{2}}\right]^{1 / 2}}\right\}=0 .
\end{aligned}
$$


As shown in references [2, 3], the factor multiplying $\sigma_{\mathrm{I}}$ in equation (22b) is non-zero for all $q$ 's, so equation (22b) implies :

$$
\sigma_{\mathrm{I}}=0
$$

and when looking for the instability threshold, we can simply set $\sigma=0$ in equation (19). The condition of neutral stability thus reads :

$\mathcal{C}=\mathscr{C}_{\mathrm{c}}\left(q^{2}\right) \equiv \frac{K}{m_{0}+K-1}+\left(1+\frac{\eta K}{m_{0}+K-1}\right) \beta q^{2}$

where $m_{0} \equiv m(\sigma=0)$.

The minimum of $\mathscr{C}_{\mathrm{c}}\left(q^{2}\right)$ in the physical region $\mathscr{C}<1$, if it exists, defines the bifurcation from the stationary planar front to a one dimensional front structure. Therefore, the position of the bifurcation is determined by the parametric equations (24) and :

$$
\beta=\frac{K}{\left(m_{0}+K-1\right)^{2}\left(2 m_{0}-1+K \eta\right)-\eta K^{2}(K-1)} .
$$

It is shown in appendix B that :

(i) If $\eta>-1$ :

- for $\beta<\beta_{\max }=\frac{1}{K(1+\eta)}, \mathscr{C}_{\mathrm{c}}\left(q^{2}\right)$ has one single minimum in the physical region;

$$
- \text { for } \beta>\beta_{\max }, \mathcal{G}_{\mathrm{c}}\left(q^{2}\right)>1 \text { for all } q \text { 's. }
$$

Therefore, for fixed $\beta<\beta_{\max }$, the system exhibits a bifurcation from the planar front state; for $\beta>\beta_{\max }$, the planar front is always linearly stable. $\beta_{\max }$ defines the "absolute stability limit». The corresponding bifurcation curve $G=G_{c}(\tilde{V})$ is drawn in figure $4 \mathrm{a}$.

(ii) If $\eta<-1: \mathscr{C}_{\mathrm{c}}\left(q^{2}\right)$ always has one single minimum in the physical region, whatever the value of $\beta$. So, in this case, there is no absolute stability limit. The shape of the corresponding bifurcation curve is represented in figure $4 \mathrm{~b}$.

Note that, for $\eta>-1$, the qualitative shape of the bifurcation curve is the same as in the dilute case. It is shown in appendix B that the effect of the " chemical capillary term " is very small in the small $\tilde{V}$ $(\beta \ll 1)$ region. It becomes important in the region $\beta \lesssim \beta_{\max }$, i.e. in the region of large critical wavelengths, where it either stabilizes or destabilizes the planar front depending on whether $\eta>0$ or $\eta<0$.

For $\eta<-1$, the chemical capillary term gives rise to a qualitative change in the shape of the instability curve : no absolute stability limit exists. $G_{\mathrm{c}}(\tilde{V})$ increases monotonously with $\tilde{V}$, and behaves like $\tilde{V}^{2}$ for large $\tilde{V}(\beta \gg 1)$.

3.2 SCALE AND SHAPE OF THE BIFURCATION CURVE FOR METALLIC AZEOTROPES. - In order to determine which kind of bifurcation should be expected for various types of quasi-azeotropic mixtures, we will

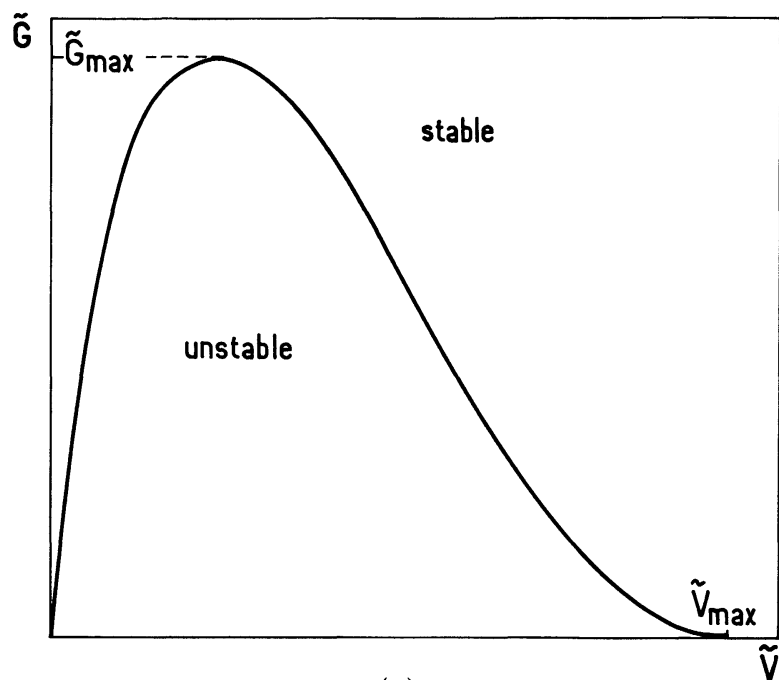

(a)

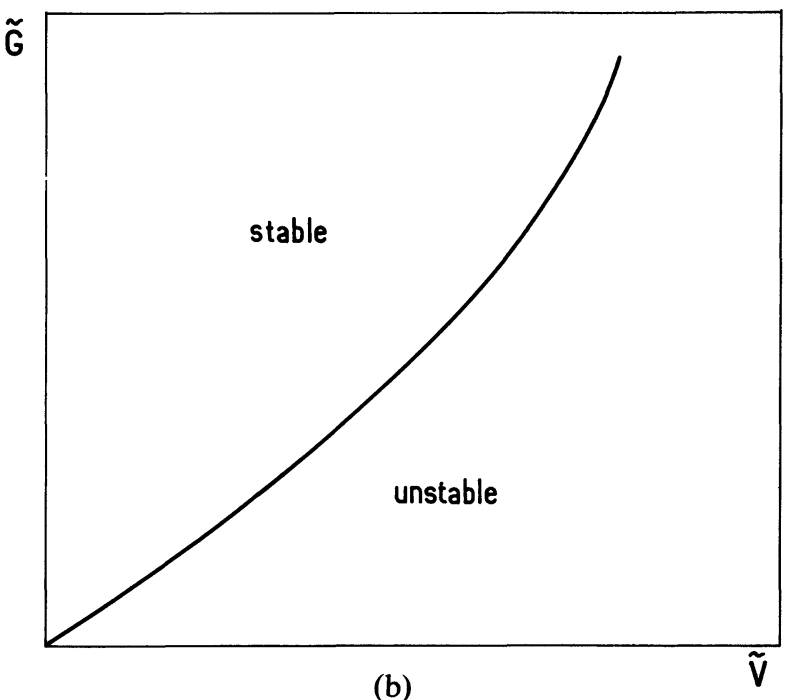

Fig. 4. - Schematic plot of the bifurcation curve in the $(\tilde{G}, \tilde{V})$ plane in the two cases $\eta>-1$ (a) and $\eta<-1$ (b) (see text).

try to estimate the order of magnitude of $\eta$. From equation (20) and equation (7) :

$\eta=\frac{2 \alpha_{\mathrm{L}}\left(\tilde{C}_{0}-1\right) \tilde{u}_{\infty}}{\tilde{T}_{0} K^{2}\left(\partial \mu_{\mathrm{S}}^{\mathbf{A}} / \partial \tilde{C}\right)}\left\{\tilde{T}_{0}\left(\Delta S^{\mathbf{B}}-\Delta S^{\mathbf{A}}\right)-\frac{\mathcal{L}}{\rho_{\mathrm{S}}}\left(\frac{\partial \rho_{\mathrm{S}}}{\partial \tilde{C}}\right)\right\}$.

In general, $\frac{1}{\rho_{\mathrm{S}}}\left|\frac{\partial \rho_{\mathrm{S}}}{\partial \tilde{C}}\right| \ll 1$. In addition, $\widetilde{T}_{0}\left|\Delta S^{\mathrm{B}}-\Delta S^{\mathrm{A}}\right|$ is roughly of the order of a latent heat of solidification, i.e. of order $\mathcal{L}$. So, the second term in the square brackets can be reasonably neglected.

The quantities $\alpha_{\mathrm{L}}, K, \widetilde{C}_{0}, \widetilde{T}_{0}$ can be obtained from the experimental determination of the equilibrium phase diagram. 
Quantities such as $S_{\mathrm{L}, \mathrm{S}}^{i}$ and $\partial \mu_{\mathrm{S}}^{\mathbf{A}} / \partial \tilde{C}$ are not known in general for real solutions. In order to calculate them, one would need a complete microscopic description. So, we will only estimate them with the help of very simple approximations.

For real solutions, one usually defines a chemical activity $a_{\phi}^{i}$ for species $i$ in phase $\phi$ by :

$$
\mu_{\phi}^{i}=\mu_{\phi}^{i 0}+\frac{R \tilde{T}_{0}}{M_{i}} \log a_{\phi}^{i}
$$

where $\mu_{\phi}^{i 0}$ is a standard chemical potential depending on temperature and pressure only, $R$ is the gas constant and $M_{i}$ is the molar mass of species $i$; the activity $a_{\phi}^{i}$ is equal to $\widetilde{C}_{i} M_{j} /\left(\widetilde{C}_{i} M_{j}+\tilde{C}_{j} M_{i}\right)$ for an ideal solution and measures, in some sense, the deviation from ideality. From activity measurements on various metallic alloys, including some which form azeotropes [8], it appears that $a_{i}^{\phi} /\left\{C_{i} M_{j} /\left(C_{i} M_{j}+C_{j} M_{i}\right)\right\}$ is a regular function of $\widetilde{C}_{i}$, which never departs noticeably from 1. So, one can safely for an order of magnitude write :

$$
\left(\partial \mu_{\mathrm{S}}^{\mathrm{A}} / \partial \tilde{C}\right) \sim R \tilde{T}_{0} / \tilde{C}_{0}\left[\tilde{C}_{0}\left(M_{\mathrm{B}}-M_{\mathrm{A}}\right)+M_{\mathrm{A}}\right] ;
$$

as in an ideal alloy.

On the other hand, in order to estimate $\Delta S^{\mathrm{B}}-\Delta S^{\mathrm{A}}$, we make use of the usual regular solution approximation [9]. That is, the change of entropy due to the formation of the solution is assumed to be equal to that for an ideal mixture. This approximation is known to be rather satisfactory for many real solutions. Then, at the azeotropic point $\left(^{4}\right): \Delta S^{\mathrm{B}}-\Delta S^{\mathrm{A}}=$ $\Delta S^{0 \mathrm{~B}}-\Delta S^{0 \mathrm{~A}}, S^{0 i}$ being the partial entropy of species $i$ in their pure states evaluated at the reference point : $\left(\widetilde{T}_{0}, \tilde{p}_{0}\right)$. Then :

$$
\Delta S^{0 i}=\frac{\mathcal{L}_{i}}{\widetilde{T}_{i 0}}+\int_{\widetilde{i}_{i 0}^{0}}^{\widetilde{T}_{0}}\left(C_{\mathrm{p}, \mathrm{L}}^{i}-C_{\mathrm{p}, \mathrm{S}}^{i}\right) \mathrm{d} \tilde{T}
$$

where $\tilde{T}_{i 0}$ and $\mathcal{L}_{i}$ are the melting temperature and latent heat of fusion at pressure $\widetilde{p}_{\mathbf{L}}$. The latent heat contribution is always much larger than the specific heat one, so that, roughly :

$$
\Delta S^{0 \mathrm{~B}}-\Delta S^{0 \mathrm{~A}} \simeq \frac{\mathfrak{L}_{\mathrm{B}}-\mathfrak{L}_{\mathrm{A}}}{\tilde{T}_{0}} .
$$

Equation (26) then becomes :

$$
\begin{aligned}
& \eta \sim \\
& -\frac{2 \alpha_{\mathrm{L}}\left(1-\tilde{C}_{0}\right)\left(\mathfrak{L}_{\mathrm{B}}-\mathfrak{L}_{\mathrm{A}}\right) \tilde{C}_{0}\left\{M_{\mathrm{A}}+\tilde{C}_{0}\left(M_{\mathrm{B}}-M_{\mathrm{A}}\right)\right\}}{\widetilde{T}_{0}^{2} K^{2} R} \tilde{u}_{\infty} .
\end{aligned}
$$

$\left({ }^{4}\right)$ This is true only at the azeotropic points, where the configurational entropics are equal in the two phases, since $\widetilde{C}_{\mathrm{S}}=\tilde{C}_{\mathrm{L}}$.
For metals $\tilde{T}_{0}$ is of order $500 \mathrm{~K}$ at least, $\alpha_{\mathrm{L}}$ is typically in the $10^{2}$ to $10^{3} \mathrm{~K}$ range and $K$ is usually of order 1 . On the other hand, for most metallic azeotropes $\tilde{C}_{0}\left|\tilde{C}_{0}\left(M_{\mathrm{B}}-M_{\mathrm{A}}\right)+M_{\mathrm{A}}\right|\left|\mathfrak{L}_{\mathrm{B}}-\mathfrak{L}_{\mathrm{A}}\right| / R \tilde{T}_{0}$ is smaller than one (the azeotropic composition is, typically, of order $50 \%$ ).

Thus :

$$
|\eta|<10^{-2}\left|\tilde{u}_{\infty}\right|
$$

with $\tilde{u}_{\infty}$ in atomic $\%$.

We have made a more precise evaluation of $\eta$ in the case of the $\mathrm{Pb}-\mathrm{Tl}$ azeotrope [10], which due to its rather low melting point, seems to be a good candidate for experiments. Using the thermodynamic quantities listed in table I, we find that $\eta \simeq+2 \times 10^{-3} \tilde{u}_{\infty}$.

So, it seems that, for metallic materials, $|\eta| \ll 1$ and the chemical capillary correction to the bifurcation diagram is, in practice, negligible. Then, as shown in $\S 3.1$, the shape of the bifurcation curve remains the same as for dilute systems. However, the scale of this curve is strongly reduced in the quasi-azeotropic situation. Indeed, from equation (25), the maximum velocity on the bifurcation diagram is given by :

$$
\beta_{\max }=\frac{1}{K(1+\eta)} \simeq \frac{1}{K}
$$

i.e.

$$
\tilde{V}_{\max } \simeq \frac{2 \alpha_{\mathrm{L}} D_{\mathrm{L}}(K-1)}{\Gamma \tilde{T}_{0} K^{3}} \tilde{u}_{\infty}^{2}
$$

where $\Gamma=\gamma / \rho_{\mathrm{S}} \mathcal{L}$ is the usual capillary length.

In the limit $\eta=0$, from equations (24), (25), one can calculate exactly the value of $G_{\max }$ :

$$
\widetilde{G}_{\max }=\frac{2(n+1) \alpha_{\mathrm{L}}^{2}(K-1)^{2}}{\Gamma \widetilde{T}_{0} K^{2}} f(K) u_{\infty}^{4}
$$

where

$$
\left.f(K)=\frac{m_{0}\left(m_{0}-1\right)}{\left(2 m_{0}-1\right)^{2}\left(m_{0}+K-1\right)^{4}}\right]_{m_{0}=\frac{1}{4}(3+\sqrt{1+8 K})}
$$

Table I. - The values of thermodynamic quantities of $\mathrm{Pb}-\mathrm{Tl}$ needed in the calculation of the coefficient $\eta$. The quantity $\partial \mu_{\mathrm{s}}^{\mathrm{Pb}} / \partial \tilde{C}$ is, with a good precision, equal to that for an ideal alloy [11].

\begin{tabular}{cc} 
Symbols & Values \\
\hline$\tilde{C}_{0}$ & $38 \%$ \\
$\widetilde{T}_{0}$ & $653 \mathrm{~K}$ \\
$\alpha_{\mathrm{L}}$ & $-7 \times 10^{2} \mathrm{~K}$ \\
$K$ & 0.7 \\
$M_{\mathrm{Pb}}$ & $207.2 \mathrm{~g}$ \\
$M_{\mathrm{T1}}$ & $204.37 \mathrm{~g}$ \\
$\mathcal{L}_{\mathrm{Pb}}$ & $6.26 \mathrm{cal} . \mathrm{g}^{-1}$ \\
$\mathcal{L}_{\mathrm{Tl}}$ & $5.04 \mathrm{cal} . \mathrm{g}^{-1}$
\end{tabular}


$\tilde{V}_{\max }$ and $\tilde{G}_{\max }$ are respectively proportional to $\tilde{u}_{\infty}^{2}$ and $\tilde{u}_{\infty}^{4}$, while in dilute mixtures they are proportional to $\tilde{u}_{\infty}$ and $\tilde{u}_{\infty}^{2}$; it is this change in the $\tilde{u}_{\infty}$ dependences of $V_{\max }$ and $\tilde{G}_{\max }$ which is responsible for the scale reduction. Typically, $D_{\mathrm{L}} \sim 10^{-5} \mathrm{~cm}^{2} / \mathrm{s}, \Gamma \tilde{T}_{0} \sim$ $10^{-5} \mathrm{~cm} . \mathrm{K}, K \sim 1, \alpha_{\mathrm{L}} \sim 10^{2}-10^{3} \mathrm{~K}$. One then finds that :

$$
\begin{array}{ll}
\tilde{V}_{\max } \sim 10^{-2} \tilde{u}_{\infty}^{2} & \text { to } 10^{-1} \tilde{u}_{\infty}^{2} \\
\tilde{G}_{\max } \sim 10 \tilde{u}_{\infty}^{4} & \text { to } 10^{3} \tilde{u}_{\infty}^{4}
\end{array}
$$

with $\tilde{V}$ in $\mathrm{cm} / \mathrm{s}, G$ in $\mathrm{K} / \mathrm{cm}$ and $\tilde{u}_{\infty}$ in atomic $\%$.

When the departure of the imposed concentration, $\tilde{C}_{\infty}$ from the azeotropic composition $\tilde{C}_{0}$ is in the atomic $\%$ range, $V_{\max }$ and $G_{\max }$ are typically reduced by factors $10^{2}$ and $10^{4}$ as compared with their values for dilute systems.

For example, for the $\mathrm{Pb}-\mathrm{T} 1$ quasi-azetropic mixture with

$$
\begin{aligned}
& \left|\tilde{u}_{\infty}\right|=1 \%, \\
& D_{\mathrm{L}} \sim 10^{-5} \mathrm{~cm} / \mathrm{s} \text { and } \tilde{T}_{0} \Gamma \sim 10^{-5} \mathrm{~cm} \cdot \mathrm{K},
\end{aligned}
$$

we obtain the following estimates :

$$
\begin{aligned}
& \tilde{V}_{\max } \sim 400 \text { to } 500 \mu / \mathrm{s} \\
& \tilde{G}_{\max } \sim 100 \text { to } 200 \mathrm{~K} / \mathrm{cm} .
\end{aligned}
$$

Such values of $\tilde{V}$ and $\tilde{G}$ are in the range accessible to actual directional solidification experiments, therefore, using quasi-azeotropic mixtures should make the complete exploration of the Mullins-Sekerka bifurcation curve possible.

3.3 THE ${ }^{3} \mathrm{He}-{ }^{4} \mathrm{He}$ AZEOTROPE. - In view of the above discussion, the question naturally arises of whether there exists any material with non negligible chemical capillarity. From equation (26), for the magnitude of $\eta$ to be significant, the material must have a small $\tilde{T}_{0}$. This leads one to consider the case of the ${ }^{3} \mathrm{He}-{ }^{4} \mathrm{He}$ azeotrope [12] at $\tilde{p}_{0}=24 \mathrm{~atm}$. (a pressure at which the phase diagram is known with good accuracy near the azeotropic point).

The specific heat measurements of Edwards et al. [13] show that ${ }^{3} \mathrm{He}-{ }^{4} \mathrm{He}$ mixtures behave, to a good approximation, as regular solutions. So, we have determined $\partial \mu_{\mathbf{S}}^{\mathbf{A}} / \partial \tilde{C}$ from the corresponding expression of the Gibbs function given in reference [14].

As mentioned in 3.2, for a regular solution, at the azeotropic point $\Delta S_{\phi}^{i}=\Delta S_{\phi}^{0 i}$. At $\widetilde{p}_{0}=24 \mathrm{~atm}$., neither ${ }^{3} \mathrm{He}$ nor ${ }^{4} \mathrm{He}$ are solid, whatever the temperature. So, in order to calculate the entropy differences of the pure species at $\widetilde{T}_{0}, \widetilde{p}_{0}$, we write :

$$
\begin{aligned}
& \Delta S^{0(3)}-\Delta S^{0(4)}= \frac{\mathfrak{L}_{3}\left(\tilde{T}_{0}\right)-\mathfrak{L}_{4}\left(\widetilde{T}_{0}\right)}{\tilde{T}_{0}}- \\
&-\int_{\tilde{p}_{0}}^{\tilde{p}_{3}} \Delta\left(\frac{\partial v_{3}}{\partial \tilde{T}}\right) \mathrm{d} \tilde{p}+\int_{\tilde{p}_{0}}^{\tilde{p}_{4}} \Delta\left(\frac{\partial v_{4}}{\partial \tilde{T}}\right) \mathrm{d} \tilde{p}
\end{aligned}
$$

where $\widetilde{p}_{i}$ is the melting pressure of the pure (i) species at $\widetilde{T}=\widetilde{T}_{0}, v_{i}$ its specific volume. $\partial v_{i} / \partial \widetilde{T}=v_{i} v_{i}$ where $v_{i}$ is the thermal expansion coefficient.

Both $v_{3}$ and $v_{4}$ vary smoothly in the pressure range $\left(\tilde{p}_{0}, \tilde{p}_{i}\right)$. So, the integral terms in the r.h.s. of equation (35) are of order $\Delta\left(\frac{\partial v_{i}}{\partial \tilde{T}}\right)\left(\tilde{p}_{i}-\tilde{p}_{0}\right)$. The values of $\tilde{p}_{i}\left(\tilde{T}_{0}\right), \mathcal{L}_{i}, v_{i}, v_{i}$ are given in table II. Since $v_{i} \sim 10^{-3} \mathrm{~K}^{-1}$, and $\mathcal{L}_{4}\left(\tilde{T}_{0}\right) \ll \mathcal{L}_{3}\left(\tilde{T}_{0}\right)$, the quantity $\Delta S^{0(3)}-\Delta S^{0(4)}$ is, to a good approximation equal to $\mathcal{L}_{3} / \widetilde{T}_{0}$.

We, then, find :

$$
\eta \simeq 0.2 \tilde{u}_{\infty} \quad\left(\tilde{u}_{\infty} \text { in atomic } \%\right) .
$$

This estimate, though rough, shows that, in the ${ }^{3} \mathrm{He}-{ }^{4} \mathrm{He}$ mixture, chemical capillarity should be non negligible.

Since $\eta$ is proportional to $\tilde{u}_{\infty}$, its sign changes with that of $\tilde{u}_{\infty}$. It follows from the analysis of the subsection 3.1 that there should be a measurable asymmetry between the bifurcation curves for the "hyper " and " hypoazeotrope", with equal $\left|\tilde{u}_{\infty}\right|$, the observation of which would permit to estimate directly the value of $\eta$.

As mentioned in $\S 3.1$, the chemical capillary effect is important in the large velocity regime $\left(\tilde{V} \sim \tilde{V}_{\max }\right)$. Thus, for the difference between the two curves to be measurable, one should work at large velocities $\tilde{V} \lesssim \tilde{V}_{\max }$. The maximum velocity is given by :

$$
\tilde{V}_{\max }^{ \pm}=\frac{2 \alpha_{\mathrm{L}} D_{\mathrm{L}}(K-1)}{\tilde{T}_{0} K^{3}(1 \pm|\eta|) \Gamma} \tilde{u}_{\infty}^{2}
$$

Table II. - The values of thermodynamic quantities of ${ }^{3} \mathrm{He}-{ }^{4} \mathrm{He}$ needed in the calculations of the coefficient $\eta$.

Symbols

Values

\begin{tabular}{ll}
\hline $\mathfrak{L}_{3}$ & $0.33 \mathrm{cal} \cdot \mathrm{g}^{-1}[16]$ \\
$v_{3 \mathrm{~L}}$ & $8.03 \mathrm{~cm}^{3} \cdot \mathrm{g}^{-1}[16]$ \\
$v_{3 \mathrm{~S}}$ & $7.67 \mathrm{~cm}^{3} \cdot \mathrm{g}^{-1}[16]$ \\
$v_{3 \mathrm{~L}}$ & $0.25 \times 10^{-3} \mathrm{~K}^{-1}[16]$ \\
$v_{3 \mathrm{~S}}$ & $4.1 \times 10^{-3} \mathrm{~K}^{-1}[16]$ \\
$\tilde{p}_{3}$ & $45.44 \mathrm{~atm} \cdot[16]$ \\
$\mathfrak{L}_{4}$ & $0.02 \mathrm{cal} . \mathrm{g}^{-1}[17]$ \\
$v_{4 \mathrm{~L}}$ & $5.77 \mathrm{~cm}^{3} / \mathrm{g}[17]$ \\
$v_{4 \mathrm{~S}}$ & $5.24 \mathrm{~cm}^{3} / \mathrm{g}[17]$ \\
$v_{4 \mathrm{~L}}$ & $-5 \times 10^{-3} \mathrm{~K}^{-1}[17]$ \\
$v_{4 \mathrm{~S}}$ & $1.1 \times 10^{-3} \mathrm{~K}^{-1}[17]$ \\
$\widetilde{p}_{4}$ & $25.18 \mathrm{~atm} \cdot[17]$ \\
$\widetilde{C}_{0}$ & $20 \%[12]$ \\
$\widetilde{T}_{0}$ & $1.2 \mathrm{~K}[12]$ \\
$\widetilde{p}_{0}$ & $24 \mathrm{~atm} \cdot[12]$ \\
$\alpha_{\mathrm{L}}$ & $-25 \mathrm{~K}[12]$ \\
$K$ & $0.5[12]$ \\
$\partial \mu_{\mathrm{S}}^{3}$ & $2.8 \mathrm{cal} . \mathrm{g}^{-1}[14]$ \\
\hline$\partial \widetilde{C}$ &
\end{tabular}


where the subscripts + and - correspond respectively to $\tilde{u}_{\infty}>0$ and $\tilde{u}_{\infty}<0$.

The latent heat of fusion $\mathcal{L} \simeq C_{0} \mathcal{L}_{3}+\left(1-\tilde{C}_{0}\right) \mathcal{L}_{4}$, the diffusion coefficient $D_{\mathrm{L}} \sim 10^{-3}$ to $10^{-4} \mathrm{~cm}^{2} / \mathrm{s}$ [15]. In the absence of measurements of the solid-liquid interface tension for ${ }^{3} \mathrm{He}-{ }^{4} \mathrm{He}$ mixtures, we take, for an order of magnitude of $\gamma$, the value measured for ${ }^{4} \mathrm{He}[18]: \gamma \sim 4 \times 10^{-9} \mathrm{cal} / \mathrm{cm}^{2}$.

We then find, for the ${ }^{3} \mathrm{He}-{ }^{4} \mathrm{He}$ azeotrope at $\tilde{p}_{0}=$ $24 \mathrm{~atm}$., and for $\tilde{u}_{\infty}=1 \%$

$$
\tilde{V}^{+} \sim 1 \text { to } 10 \mathrm{~cm} / \mathrm{s}
$$

with $\tilde{V}^{-} / \tilde{V}^{+1} \simeq 1.5$.

Growth velocities of this order of magnitude have already been reached in solidification experiments on pure ${ }^{4} \mathrm{He}$ [19]. So, since the ratio $\tilde{V}^{-} / \tilde{V}^{+}$is significant (due to the importance of $\eta$ ), the ${ }^{3} \mathrm{He}-{ }^{4} \mathrm{He}$ azeotrope seems to be a good candidate on which to perform experiments and measure the effect of the chemical capillary term.

\section{Non linear stability analysis.}

We now want to study the nature of the bifurcation. That is, we need to perform a non-linear analysis up to third order in the amplitude of front deformations [2].

For this purpose, we follow the approach of Wollkind and Segel [2]. That is, we look for solutions of equations $((3)-(5))$ of the form :

$$
\begin{gathered}
\mathbf{v}(x, z, t)=\sum_{n=0}^{\infty} \varepsilon^{n} \mathbf{v}_{n}(x, z, t) \\
\varepsilon \xi(x, t)=\sum_{n=1}^{\infty} \varepsilon^{n} \xi_{n}(x, t)
\end{gathered}
$$

and assume that :

$$
\begin{aligned}
\mathbf{v}_{1}(x, z, t) & =A(t) \cos (q x) \mathbf{v}_{1}(z) \\
\varepsilon \xi_{1}(x, t) & =A(t) \cos (q x)
\end{aligned}
$$

where $\mathbf{v}_{1}(z)$ is the solution of the linearized problem.

Inserting equations (39), (40) into the basic equations, one straigthforwardly deduces that :

$$
\begin{aligned}
& \mathbf{v}_{2}(x, z, t)=A^{2}(t)\left[\mathbf{v}_{20}(z)+\mathbf{v}_{22}(z) \cos (2 q x)\right] \\
& \mathbf{v}_{3}(x, z, t)= \\
& \quad=A^{3}(t)\left[\mathbf{v}_{31}(z) \cos (q x)+\mathbf{v}_{33}(z) \cos (3 q x)\right]
\end{aligned}
$$

etc...

From this one easily [2] checks that the time dependent amplitude $A(t)$ of mode $q$ must satisfy an equation of the form :

$$
\frac{\mathrm{d} A}{\mathrm{~d} t}=\sigma A-\varepsilon^{2} a_{1} A^{3}+\mathrm{O}\left(\varepsilon^{3}\right)
$$

where $\sigma$ is the relaxation rate of mode $q$, given by the dispersion equation (19).

The Landau constant $a_{1}$, the sign of which determines the nature of the bifurcation, will now be calculated from the full third order expansion.

Following Caroli et al. [3], the solutions $\mathbf{v}_{20}, \mathbf{v}_{22}$ of the second order problem are (in the « small thermal box » case, we consider here)

$$
\begin{aligned}
& T_{\mathrm{L}}^{20}=\alpha_{20}\left(z-L_{1}\right) \\
& T_{\mathrm{L}}^{22}=\alpha_{22} \mathrm{e}^{-2 q z} \\
& T_{\mathrm{S}}^{20}=\alpha_{20}^{\prime}\left(z+L_{2}\right) \\
& T_{\mathrm{S}}^{22}=\alpha_{22}^{\prime} \mathrm{e}^{2 q z} \\
& u_{\mathrm{L}}^{20}=\beta_{20} \mathrm{e}^{-h z} \\
& u_{\mathrm{L}}^{22}=\beta_{22} \mathrm{e}^{-h^{\prime} z}
\end{aligned}
$$

where

$h=\frac{1}{2}+\sqrt{\frac{1}{4}+2 \sigma} ; \quad h^{\prime}=\frac{1}{2}+\sqrt{\frac{1}{4}+2 \sigma+4 q^{2}}$.

The values of $\alpha_{20}, \ldots, \beta_{22}$, obtained from the second order expansion of the interface equations, are listed in appendix $\mathrm{C}$.

In order to calculate $a_{1}$, we only need to calculate the $v_{31}$ terms. They are given by $[2,3]$ :

$T_{\mathrm{L}}^{31}(z)=\alpha_{31} \mathrm{e}^{-q z}$

$T_{\mathrm{S}}^{31}(z)=\alpha_{31}^{\prime} \mathrm{e}^{q z}$

$u_{\mathrm{L}}^{31}(z)=\beta_{31} \mathrm{e}^{-h^{\prime \prime} z}+\frac{\beta_{1} a_{1}}{2 \sigma}\left[\mathrm{e}^{-m z}-\mathrm{e}^{-h^{\prime \prime} z}\right]$

where $h^{\prime \prime}=\frac{1}{2}+\sqrt{q^{2}+\frac{1}{4}+3 \sigma}$.

The interface boundary conditions (4) and (6) lead, in the limit $\sigma=0$, to the following system :

$$
\begin{gathered}
\alpha_{31}-\alpha_{31}^{\prime}+\frac{G(n-1)}{n} \xi_{31}=d_{1} \\
n \alpha_{31}^{\prime}+\alpha_{31}=d_{2}
\end{gathered}
$$

$\left(1-K-m_{0}\right) \beta_{31}+\left[K(1-K)+d_{0}^{\mathrm{c}} q^{2}\right] \xi_{31}=$

$$
\begin{aligned}
d_{3}-a_{1}\left[K-1+\frac{\beta_{11}}{2 m_{0}-1}\right] & \\
\alpha_{31}+[G-2 M(K-1)+ & \left.d_{0} q^{2}\right] \xi_{31} \\
& -2 M \beta_{31}=d_{4} .
\end{aligned}
$$

The values of $d_{i}$ are listed in appendix $C$.

One then easily checks that the determinant of the system (45) is identical to that of the linearized system (18) for $\sigma=0$, which precisely vanishes at the bifurcation.

One, therefore, must write a condition of compatibi- 
lity for equations (45), which reads :

$$
\begin{aligned}
a_{1} & =\frac{2 m_{0}-1}{(K-1)\left(2 m_{0}-1-\ell+\beta q^{2}\right)} \times \\
& \times\left\{d_{3}-\frac{m_{0}+K-1}{2}\left[d_{4}-\frac{d_{2}+n d_{1}}{n+1}\right]\right\}
\end{aligned}
$$

In equation (46), $\mathcal{C}, \beta, q$ (and $m_{0}$ ) are understood to be evaluated at the bifurcation. They are related to each other by the parametric equations (24)-(25). Thus, $a_{1}$ is in fact a function of a single variable, for instance $\beta$ (i.e. of the critical pulling velocity).

However, due to the number of independent parameters $\left(K, \eta, n, L_{1} / L_{2}\right)$ involved in expression (46), it would be unfeasibly heavy to try to scan the complete parameter space numerically.

In the absence of experiments on azeotropic systems, we have performed illustrative numerical analysis of the variation of $a_{1}$. For this purpose, we choose values for $L_{1} / L_{2}, \eta, n$, which are plausible for experiments on metallic azeotropes. That is :

(i) on the basis of the estimates performed in 3.2, we neglect chemical capillarity, i.e. take $\eta=0$;

(ii) we take for the thermal conductivity ratio $n$, the typical value $n=2$;

(iii) we assume that the experimental set up is adjusted so that $L_{1}=L_{2}$. We have checked numerically that changing $L_{1} / L_{2}$ by a factor 2 only induces negligible variation of $a_{1}$.

Under these assumptions, we have computed $a_{1}$, as a function of $\tilde{V}$ (where $\tilde{V}$ runs along the bifurcation curve) for various values of the distribution coefficient $K$. Positive $a_{1}$ 's (resp. negative $a_{1}$ 's) correspond to a normal (resp. subcritical) bifurcation [2]. We list in table III, for different values of $K$, the range of values of $\tilde{V} / \tilde{V}_{\max }$ in which the bifurcation should be normal

Table III. - Numerical values of Landau's constant $a_{1}$.

\begin{tabular}{ll}
\multicolumn{1}{c}{$K$} & Range of $\frac{\tilde{V}}{\tilde{V}_{\text {max }}}$ for which $a_{1}>0$ \\
\hline 0 to 0.63 & none \\
0.64 & $1.45-1.55$ \\
0.65 & $1.37-1.52$ \\
0.7 & $0.60-1$ \\
0.8 & $0.2-1$ \\
0.9 & $0.8 \times 10^{-1}-1$ \\
1.1 & $0.26 \times 10^{-1}-1$ \\
1.2 & $0.16 \times 10^{-1}-1$ \\
1.3 & $0.115 \times 10^{-3}-1$ \\
1.4 & $0.83 \times 10^{-2}-1$ \\
1.5 & $0.56 \times 10^{-2}-1$ \\
1.6 & $0.33 \times 10^{-2}-1$ \\
1.7 & $0.20 \times 10^{-2}-1$ \\
1.8 & $0.18 \times 10^{-3}-1$ \\
1.9 & $0.0-1$ \\
2 & $0.0-1$
\end{tabular}

$\left(a_{1}>0\right)$. It is seen that, for $K<0.64$, the bifurcation is inverted everywhere along the instability diagram. When $K$ increases above this value, normal bifurcation range develop on the large $\tilde{V}$ side of the diagram, and extends very rapidly towards low $\tilde{V}$ 's; for $K \gtrsim 1.5$, the bifurcation should be normal practically everywhere. Note that the most frequent situation for metallic azeotropes correspond to values of $K$ larger than 1 [20].

\section{Conclusion.}

Our major results can be summarized as follows :

(i) The quadratic shape of the phase diagram of the binary mixture around an azeotropic point gives rise to an important reduction of the scale of the MullinsSekerka bifurcation diagram. Our estimates indicates that directional solidification experiments should be able to explore this diagram completely. In particular, it would be interesting to check whether the high velocity front state (in the "restabilized" region) depends or not on the path followed by the system in the $\tilde{G}, \tilde{V}$ plane.

(ii) For not too small values of the distribution coefficient $K$ (typically $K>0.7$ ), the normal bifurcation regime should become accessible. As discussed in $\S 1$, such experiments would provide very valuable informations to guide further progress in the understanding of the shape of cellular fronts. It might even be possible to find materials in which, by moving along the bifurcation curve, one could compare front shapes close above threshold in the normal and subcritical regimes.

(iii) As discussed in $\S 3.3$, the chemical capillary correction is very likely negligible in practice for ordinary azeotropes. However, it should be of importance in the ${ }^{3} \mathrm{He}-{ }^{4} \mathrm{He}$ case, where it might give rise to a noticeable difference between the bifurcations curves for systems with opposite values of $\tilde{C}_{\infty}-\tilde{C}_{0}$.

Finally, it may be worth mentioning that being able to explore the upper part $\left(\tilde{V}^{*} \sim \vec{V}_{\max }\right)$ of the bifurcation curve would give access to the "boundary-layer regime » (21), in which the critical wavelength becomes much larger than the diffusion length. Indeed, as can be seen from equation (25), $q_{\mathrm{c}} \rightarrow 0$ for $\tilde{V} \rightarrow \tilde{V}_{\max }\left(q_{\mathrm{c}}\right.$ is the critical wavenumber). Experiments in this regime would be useful to check the semi-empirical local model - analogous to that built up by Ben Jacob et al. [21] for dendritic growth in an undercooled meltrecently proposed by Karma and Goldenfeld [22].

Note, however, that, in the immediate vicinity of $\tilde{V}_{\text {max }}$, our linear stability calculation should be extended to include the reflection of temperature fluctuations on the external boundaries. It may be checked that this effect might be non negligible for $\left(\tilde{V}_{\max }-\right.$ $\tilde{V}) / \tilde{V}_{\max } \lesssim 10^{-2}$.

Moreover, in this regime, our amplitude expansion ceases to be valid. Indeed, since $q_{\mathrm{c}} \rightarrow 0$, harmonics of the fundamental mode $q_{\mathrm{c}}$ are quasi-neutral, and their 
contribution can no longer be neglected. So, one should either resort to the semi-empirical boundary - layer approach or develop a singular expansion analogous to the one performed by Sivashinsky [23] for dilute systems with very small segregation coefficients.

So, it seems, from our calculations, that quasiazeotropic mixtures should give access to a wider range of physical behaviours than is the case for dilute mixtures. Therefore, they appear as very interesting systems on which to perform systematic directional solidification experiments.

\section{Acknowledgments.}

It is a pleasure to acknowledge a very fruitful collaboration and discussions with my advisors Christiane Caroli, Bernard Roulet and Bernard Caroli. I would like to thank Sebastien Balibar and François Gallet for helpful informations and discussions abbut helium.

\section{Appendix A.}

In this appendix, we derive the conditions of chemical equilibrium at the interface (Eqs. (6a, b)). Let $\mu_{\mathrm{L}, \mathrm{s}}^{i}$ be the chemical potential of species $i(i=\mathrm{A}, \mathrm{B})$ in the liquid or solid phase. Local chemical equilibrium on the interface implies :

$$
\mu_{\mathrm{L}}^{i}\left(\tilde{T}_{\mathrm{L}}, \tilde{p}_{\mathrm{L}}, \tilde{C}_{\mathrm{L}}\right)=\mu_{\mathrm{S}}^{i}\left(\tilde{T}_{\mathrm{S}}, \tilde{p}_{\mathrm{S}}, \tilde{C}_{\mathrm{S}}\right)
$$

where $\widetilde{p}_{\mathrm{L}, \mathrm{S}}$ is the pressure on the liquid or solid side of the interface.

We expand equation (A.1) about the reference state $\left(\tilde{T}_{0}, \tilde{p}_{0}, \tilde{C}_{0}\right)$ where we choose for $\tilde{p}_{0}$ the fixed liquid pressure $\widetilde{p}_{\mathrm{L}}$ and $\widetilde{T}_{0}, \widetilde{C}_{0}$ are the temperature and concentration of the azeotropic point at pressure $\tilde{p}_{0}$ (they correspond to equilibrium between solid and liquid with a planar interface). To first order in $\delta \tilde{T}=\tilde{T}-\tilde{T}_{0}$ and $\delta \tilde{p}_{\mathrm{S}} \equiv \tilde{p}_{\mathrm{S}}-\tilde{p}_{0}$, and to second $\operatorname{order}\left({ }^{5}\right)$ in $\delta \widetilde{C}^{0} \equiv \widetilde{C}-\widetilde{C}_{0}$, we obtain :

$$
\begin{aligned}
\Delta S^{i} \delta \tilde{T}-r_{\mathrm{L}}^{i} \delta \tilde{C}_{\mathrm{L}}+r_{\mathrm{S}}^{i} \delta \tilde{C}_{\mathrm{S}}- \\
\quad-\lambda_{\mathrm{L}}^{i} \delta \tilde{C}_{\mathrm{L}}^{2}+\lambda_{\mathrm{S}}^{i} \delta \tilde{C}_{\mathrm{S}}^{2}+v_{\mathrm{S}}^{i} \gamma \tilde{\kappa}=0
\end{aligned}
$$

where $\Delta S^{i} \equiv S_{\mathrm{L}}^{i}-S_{\mathrm{S}}^{i} ;$ with $S_{\mathrm{L}, \mathrm{S}}^{i}=-\partial \mu_{\mathrm{L}, \mathrm{S}}^{i} / \partial \tilde{T}_{\mathrm{L}, \mathrm{S}}$

$$
\begin{aligned}
& r_{\mathrm{L}, \mathbf{S}}^{i} \equiv \partial \mu_{\mathrm{L}, \mathbf{S}}^{i} / \partial \tilde{C}_{\mathbf{L}, \mathbf{S}} ; \quad \lambda_{\mathbf{L}, \mathbf{S}}^{i} \equiv \frac{1}{2} \partial^{2} \mu_{\mathbf{L}, \mathbf{S}}^{i} / \partial \tilde{C}_{\mathbf{L}, \mathbf{S}}^{2} \\
& v_{\mathbf{S}}^{i}=\partial \mu_{\mathbf{S}}^{i} / \partial \tilde{p}_{\mathbf{S}}
\end{aligned}
$$

and where we have made use of Laplace's law : $\tilde{p}_{\mathrm{S}}-$ $\tilde{p}_{\mathrm{L}}=\gamma \tilde{\kappa} ; \gamma$ is the interfacial tension and $\tilde{\kappa}$ the surface curvature defined as positive for a convex solid.

$\left({ }^{5}\right)$ Due to the fact that $\delta \tilde{T} \sim \delta \tilde{C}^{2}$ at equilibrium, one has to expand $\mu_{\mathrm{L}, \mathrm{s}}^{i}$ up to second order in $\delta \tilde{C}$.
One easily checks that the two equations (A.2) are equivalent to :

$$
\begin{aligned}
& \left(r_{\mathrm{L}}^{\mathrm{A}} \Delta S^{\mathrm{B}}-r_{\mathrm{L}}^{\mathrm{B}} \Delta S^{\mathrm{A}}\right) \delta \tilde{C}_{\mathrm{L}}+\left(\lambda_{\mathrm{L}}^{\mathrm{A}} \Delta S^{\mathrm{B}}-\lambda_{\mathrm{L}}^{\mathrm{B}} \Delta S^{\mathrm{A}}\right) \delta \tilde{C}_{\mathrm{L}}^{2} \\
& -\left(r_{\mathrm{S}}^{\mathrm{A}} \Delta S^{\mathrm{B}}-r_{\mathrm{S}}^{\mathrm{B}} \Delta S^{\mathrm{A}}\right) \delta \tilde{C}_{\mathrm{S}}-\left(\lambda_{\mathrm{S}}^{\mathrm{A}} \Delta S^{\mathbf{B}}-\lambda_{\mathrm{S}}^{\mathrm{B}} \Delta S^{\mathrm{A}}\right) \delta \tilde{C}_{\mathrm{S}}^{2} \\
& -\gamma \tilde{\mathcal{K}}\left(v_{\mathbf{S}}^{\mathbf{A}} \Delta S^{\mathbf{B}}-v_{\mathbf{S}}^{\mathbf{B}} \Delta S^{\mathbf{A}}\right)=0 \\
& \left(r_{\mathrm{S}}^{\mathrm{B}} \Delta S^{\mathrm{A}}-r_{\mathrm{S}}^{\mathbf{A}} \Delta S^{\mathbf{B}}\right) \delta \widetilde{T}=\left(\lambda_{\mathrm{L}}^{\mathrm{A}} r_{\mathrm{S}}^{\mathrm{B}}-\lambda_{\mathrm{L}}^{\mathbf{B}} r_{\mathrm{S}}^{\mathrm{A}}\right) \delta \widetilde{C}_{\mathrm{L}}^{2} \\
& +\left(\lambda_{\mathrm{S}}^{\mathrm{B}} r_{\mathrm{S}}^{\mathrm{A}}-\lambda_{\mathrm{S}}^{\mathbf{A}} r_{\mathrm{S}}^{\mathbf{B}}\right) \delta \tilde{C}_{\mathrm{S}}^{2}-\left(v_{\mathrm{S}}^{\mathbf{A}} r_{\mathrm{S}}^{\mathbf{B}}-v_{\mathrm{S}}^{\mathbf{B}} r_{\mathrm{S}}^{\mathrm{A}}\right) \gamma \tilde{\kappa}
\end{aligned}
$$

where we have used the Gibbs-Duhem conditions :

$$
\tilde{C}_{0} r_{\mathrm{L}, \mathrm{S}}^{\mathrm{A}}+\left(1-\tilde{C}_{0}\right) r_{\mathrm{L}, \mathrm{S}}^{\mathrm{B}}=0 \text {. }
$$

For a planar front, near the azeotropic point, to lowest order in $\delta \tilde{C}_{\mathrm{L}, \mathrm{S}}$ :

$$
\begin{aligned}
\delta \tilde{T} & =\alpha_{\mathbf{L}} \delta \tilde{C}_{\mathbf{L}}^{2} \\
& =\alpha_{\mathbf{S}} \delta \tilde{C}_{\mathbf{S}}^{2} ; \text { with } \quad \alpha_{\mathrm{L}, \mathbf{S}} \equiv \frac{1}{2}\left[\partial^{2} \tilde{T}_{\mathrm{L}, \mathbf{S}} / \partial \tilde{C}_{\mathrm{L}, \mathbf{S}}^{2}\right]_{\tilde{c}=\tilde{c}_{0}}
\end{aligned}
$$

Equations (A .5), (A .6) must hold for a planar interface $(\tilde{\kappa}=0)$. This entails :

$$
\begin{gathered}
r_{\mathrm{S}}^{\mathrm{B}} \Delta S^{\mathrm{A}}-r_{\mathrm{S}}^{\mathrm{A}} \Delta S^{\mathrm{B}}=\frac{1}{\alpha_{\mathrm{L}}}\left(\lambda_{\mathrm{L}}^{\mathrm{A}} r_{\mathrm{S}}^{\mathrm{B}}-\lambda_{\mathrm{L}}^{\mathrm{B}} r_{\mathrm{S}}^{\mathrm{A}}\right)+ \\
+\frac{1}{\alpha_{\mathrm{S}}}\left(\lambda_{\mathrm{S}}^{\mathrm{B}} r_{\mathrm{S}}^{\mathrm{A}}-\lambda_{\mathrm{S}}^{\mathrm{A}} r_{\mathrm{S}}^{\mathrm{B}}\right) \\
K \equiv \sqrt{\frac{\alpha_{L}}{\alpha_{\mathrm{S}}}}=\frac{r_{\mathrm{L}}^{\mathrm{A}} \Delta S^{\mathrm{B}}-r_{\mathrm{L}}^{\mathrm{B}} \Delta S^{\mathrm{A}}}{r_{\mathrm{S}}^{\mathrm{A}} \Delta S^{\mathrm{B}}-r_{\mathrm{S}}^{\mathbf{B}} \Delta S^{\mathrm{A}}}
\end{gathered}
$$

For a curved interface, one then gets from (A.4) :

$$
\delta \tilde{C}_{\mathrm{S}}=K \delta \tilde{C}_{\mathrm{L}}-\frac{v_{\mathrm{S}}^{\mathbf{A}} \Delta S^{\mathrm{B}}-v_{\mathrm{S}}^{\mathbf{B}} \Delta S^{\mathbf{A}}}{r_{\mathrm{S}}^{\mathbf{A}} \Delta S^{\mathbf{B}}-r_{\mathrm{S}}^{\mathbf{B}} \Delta S^{\mathbf{A}}} \gamma \tilde{\kappa}+\mathrm{O}_{2}\left(\delta \tilde{C}_{\mathrm{L}}\right)
$$

Inserting equation(A.9) into equation(A.5) neglecting higher order terms (e.g. $\sim \widetilde{\kappa} \delta \widetilde{C})$ and using equations (A.8), we obtain :

$$
\delta \tilde{T}=\alpha_{\mathrm{L}} \delta C^{2}-\frac{r_{\mathrm{S}}^{\mathbf{B}} v_{\mathrm{S}}^{\mathbf{A}}-r_{\mathrm{S}}^{\mathbf{A}} v_{\mathrm{S}}^{\mathbf{B}}}{r_{\mathrm{S}}^{\mathbf{B}} \Delta S^{\mathbf{A}}-r_{\mathrm{S}}^{\mathbf{A}} \Delta S^{\mathbf{B}}} \gamma \tilde{\kappa} .
$$

We now want to express $\delta \tilde{T}$ and $\delta \widetilde{C}_{\mathrm{s}}$ in terms of more usual thermodynamic quantities. For that purpose, we make use of the fact that the partial entropy density $S^{i}$ relative to species $i$ is related to the total entropy density (per unit mass) $S$ by Euler's theorem (Eq. (8)). Analogous expressions hold for the densities of all extensive quantities, e.g. the specific volume.

This allows us to rewrite equations (A.9), (A.10) 
as follows :

$$
\begin{aligned}
\delta \tilde{C}_{\mathrm{S}}=K \delta \tilde{C}_{\mathrm{L}}+\frac{\left(\tilde{C}_{0}-1\right) \gamma \tilde{\kappa}}{\mathcal{L} r_{\mathrm{S}}^{\mathrm{A}} \rho_{\mathrm{S}}} \times \\
\times\left[\tilde{T}_{0}\left(\Delta S^{\mathrm{B}}-\Delta S^{\mathrm{A}}\right)-\left(\frac{\partial \rho_{\mathrm{S}}}{\partial \tilde{C}_{\mathrm{S}}}\right) \frac{\mathcal{L}}{\rho_{\mathrm{S}}}\right] \\
\delta \tilde{T}=\alpha_{\mathrm{L}} \delta \tilde{C}_{\mathrm{L}}^{2}-\frac{\tilde{T}_{0} \gamma \tilde{\kappa}}{\mathcal{L} \rho_{\mathrm{S}}}
\end{aligned}
$$

where $\mathcal{L}$ is the latent heat of fusion per unit mass and $\rho_{\mathrm{S}}$ is the solid density. Equations (A.11), (A.12) can be reduced to non dimensional forms, they then reduce to expressions $(6 a, b)$.

\section{Appendix B.}

The bifurcation is defined by the parametric equations (24), (25). For a given $\beta$, the minimum of $\mathcal{G}_{\mathrm{c}}\left(q^{2}\right)$ (if it exists) in the physical region $\mathcal{C}<1$ defines the bifurcation from the planar to a deformed front state. The condition for the existence of a minimum is expressed by equation (24), which can be rewritten :

$$
\begin{array}{r}
K / \beta=\left(m_{0}+K-1\right)^{2}\left(2 m_{0}-1+K \eta\right)- \\
-\eta K^{2}(K-1) \equiv g\left(m_{0}\right)
\end{array}
$$

with

$$
m_{0}=\frac{1}{2}+\sqrt{q^{2}+\frac{1}{4}} \geqslant 1
$$

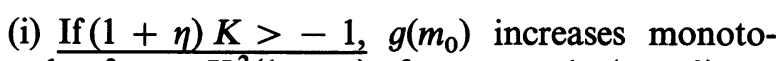
nously from $K^{2}(1+\eta)$ for $m_{0}=1 \quad(q=0)$ to $+\infty$ for $m_{0} \rightarrow+\infty(q \rightarrow \infty)$. So :

- if $(1+\eta)>0$, equation (B.1) has one single solution for $\beta<1 /(K(1+\eta))$ and no solution for $\beta>1 / K(1+\eta)$.
- if $(1+\eta)<0$, equation (B.1) always has one solution.

(ii) If $(1+\eta) K<-1, g\left(m_{0}\right)$ has one minimum for $m_{0}=m_{0 \min }=[2-K(1+\eta)] / 3>1$. Since $g\left(m_{0}=1\right)=K^{2}(1+\eta)<0, g\left(m_{0 \min }\right)<0$. That is $g\left(m_{0}\right)$ increases monotonously in the region where it is positive, and since $K / \beta>0$, equation (B.1) always has one single solution.

One can restate the above results under the following form :

(i) If $\eta>-1$;

- for $\beta<1 /[K(1+\eta)], \mathscr{C}_{\mathrm{c}}\left(q^{2}\right)$ has one single minimum in the physical region;

- for $\beta>1 /[K(1+\eta)], \mathfrak{C}_{\mathrm{c}}\left(q^{2}\right)>1$ for all $q$ 's. In this case, there is no bifurcation, the planar front is linearly stable at all $\mathcal{C}^{\prime}$ s (i.e. at all thermal gradients). $\beta_{\max } \equiv 1 /[K(1+\eta)]$ is the limit of " absolute stability $"$.

(ii) If $\eta<-1: \mathscr{C}_{\mathrm{c}}\left(q^{2}\right)$ always has one single minimum in the physical region whatever the value of $\beta$.

We will now study rapidly the shape of the bifurcation curve in the two cases $\eta>-1$ and $\eta<-1$.

(i) $\eta>-1: \beta$ varies in the range $\left(0, \beta_{\max }\right)$. The dimensionless critical wavenumber $q_{\mathrm{c}}(\beta)$ given by equation (B.1) verifies :

$$
q_{\mathrm{c}}(0)=\infty ; \quad q_{\mathrm{c}}\left(\beta_{\max }\right)=0 .
$$

For small $\tilde{V}$ 's $\left(\beta \ll 1, q_{\mathrm{c}} \gg 1\right)$ one gets, from a Taylor expansion of equations (24), (25) and by using definitions (20) of $\mathcal{G}$ and $\beta$, that :

$$
G_{c}(\tilde{V})=\frac{(n+1)(K-1) \alpha_{\mathrm{L}} \tilde{u}_{\infty}^{2}}{K^{2} D_{\mathrm{L}}} \tilde{V}+\mathrm{O}_{4 / 3}(\tilde{V})
$$

Note that the coefficient of $\tilde{V}$ in the r.h.s. of (B.3) is $\eta$-independent. This means that the chemical capillary effect is negligible in the small $\tilde{V}$ regime. For large $\tilde{V}\left(\tilde{V} \sim \tilde{V}_{\max } q_{\mathrm{c}} \ll 1\right)$ one finds :

$$
G_{c}(\tilde{V})=\frac{\Gamma \tilde{T}_{0} K(n+1)(\eta+1)^{2}[7 K(1+\eta)+4]\left(\tilde{V}-\tilde{V}_{\max }\right)^{2}}{32 D_{\mathrm{L}}^{2}[1+K(1+\eta)]^{2}}+O_{3}\left(\tilde{V}_{\max }-\tilde{V}\right)
$$

$\Gamma \equiv \gamma\left(\mathcal{L} \rho_{\mathrm{S}}\right)^{-1}$ is the usual capillary length.

From equations (24) and the definition of $\mathcal{C}$ (Eq. (20)), one easily shows that $\mathrm{d} \widetilde{G}_{\mathrm{c}} / \mathrm{d} \tilde{V}$ vanishes if and only if :

$$
\left(m_{0}+K-1\right)\left[2 m_{0}^{2}+m_{0}(K \eta-3)-K(1+\eta)+1\right]=K^{2} \eta m_{0} .
$$

This equation has one solution in the physical region $m_{0}>1$. That is to say $G_{c}(\tilde{V})$ has a single maximum.

In the limit $\eta=0$, the solution of equation (B.5) is $m_{0}=\frac{1}{4}(3+\sqrt{1+8 K})$. The corresponding value of $\tilde{V}$ is given by :

$$
\tilde{V}_{\mathrm{m}}=\frac{64 D_{\mathrm{L}}(K-1) \alpha_{\mathrm{L}} \tilde{u}_{\infty}^{2}}{\Gamma \tilde{T}_{0} K(4 K-1+\sqrt{1+8 K})^{2}(1+\sqrt{1+8 K})}
$$


and the maximum thermal gradient $G_{\max }$ is given by :

$$
G_{\max }=\frac{2(n+1) \alpha_{L}^{2}(K-1)^{2}}{\Gamma \widetilde{T}_{0} K^{2}} f(K) \tilde{u}_{\infty}^{4}
$$

with

$$
\left.f(K) \equiv \frac{m_{0}\left(m_{0}-1\right)}{\left(2 m_{0}-1\right)^{2}\left(m_{0}+K-1\right)^{4}}\right]_{m_{0}=\frac{1}{4}(3+\sqrt{1+8 K})}
$$

(ii) $\underline{\eta<-1}: \beta$ varies from 0 to $\infty$. The critical wavenumber $q_{\mathrm{c}}(\beta)$ is infinite for $\beta=0$ and has a finite value for $\beta=\infty$.

For small $\tilde{V}, G_{c}(\tilde{V})$ is given by equation (B.3). In the large $\tilde{V}$ regime $(\tilde{V} \rightarrow \infty)$, one checks from the parametric equations (24), (25) that $\tilde{G}_{\mathrm{c}}(\bar{V}) \propto \tilde{V}^{2}$. In this case, equation (B.5) has no solution with $m_{0}>1$, i.e. $G_{\mathrm{c}}(\tilde{V})$ increases monotonously with $\tilde{V}$.

\section{Appendix C.}

We list here the various amplitudes appearing in the first and second order contributions to the temperature and concentration fields.

One finds to first order (Eqs. (17)) :

$$
\begin{aligned}
& \alpha_{1}=\frac{1-n}{1+n} G \\
& \alpha_{1}^{\prime}=\frac{n-1}{n(n+1)} G \\
& \beta_{1}=(1-K)\left(C-\beta q^{2}\right) .
\end{aligned}
$$

The amplitudes of the second order terms (Eqs. (43)) are at the bifurcation :

$$
\begin{aligned}
& \alpha_{20}=\frac{(n-1)(K-1)(1-\mathfrak{C})}{2\left(n L_{1}+L_{2}\right)}\left[q(n-1)+2(n+1) \xi_{20}\right] \\
& \alpha_{20}^{\prime}=\frac{\alpha_{20}}{n} \\
& \beta_{20}=(K-1)\left[\frac{1}{4}-\xi_{20}-\frac{m_{0}\left(\mathcal{C}-\beta q^{2}\right)}{2}\right] \\
& \alpha_{22}=\frac{(1-n)(K-1)(1-\mathfrak{C})\left(q+2 \xi_{22}\right)}{2} \\
& \alpha_{22}^{\prime}=\frac{(1-n)(K-1)(1-\mathfrak{C})\left(q-2 \xi_{22}\right)}{2 n} \\
& \beta_{22}=K(K-1)\left[\frac{1}{4}-\frac{1}{2}\left(\mathcal{C}-\beta q^{2}\right)\left(m_{0}+\frac{2 q^{2}}{K}\right)-\left(1+4 \eta \beta q^{2}\right) \xi_{22}\right] /\left(h_{0}^{\prime}+K-1\right)
\end{aligned}
$$

with :

$$
\begin{aligned}
& \xi_{20}=\frac{1}{2(n+1)}\left[q(1-n)+\frac{(K-1)\left(1+n L_{1} / L_{2}\right)\left(\mathscr{C}-\beta q^{2}-1\right)^{2}}{(1-\mathfrak{C})\left(1+L_{1} / L_{2}\right)}\right] \\
& \xi_{22}=\left\{\left[K-2+\left(\mathscr{C}-\beta q^{2}\right)\left\{(K-1)\left(\mathscr{C}-\beta q^{2}\right)+2\left(m_{0}-K+1\right)\right\}\right]\left(h_{0}^{\prime}+K-1\right)+\right. \\
& \left.\quad+K-2\left(\mathscr{C}-\beta q^{2}\right)\left(m_{0} K+2 q^{2}\right)\right\} /\left\{4\left(4 \beta q^{2}\left[h_{0}^{\prime}+K(1+\eta)-1\right]+K-\mathscr{C}\left(h_{0}^{\prime}+K-1\right)\right)\right\} \\
& \text { where } h_{0}^{\prime} \equiv h^{\prime}(\sigma=0), \text { and } h_{0}=h(\sigma=0) .
\end{aligned}
$$


Finally, in the limit $\sigma=0$, the coefficients $d_{i}$ appearing in the r.h.s. of equations (45) are given by :

$$
\begin{aligned}
& d_{1}=\frac{(n-1)(K-1)(1-6)}{2 n}\left[(1-n)\left(q^{2}+3 q \xi_{22}+2 q \xi_{20}+\frac{q^{2}}{4}(3 n-5)\right]\right. \\
& d_{2}=2 q(1-n)(K-1)(1-\mathcal{C})\left(\xi_{20}+\frac{\xi_{22}}{2}\right) \\
& d_{3}=\frac{K(K-1)}{8}\left(1-3 \eta \beta q^{2}\right)+\beta_{1} m_{0}^{2} \frac{\left(m_{0}+3 K-1\right)}{8}-K \beta_{20}+ \\
& +\beta_{22}\left[q^{2}-\frac{h_{0}^{\prime 2}}{2}-\frac{h_{0}^{\prime}}{2}(K-1)\right]+\xi_{20}\left[K(1-K)-m_{0} \beta_{1}\left(m_{0}+K-1\right)\right] \\
& +\frac{\xi_{22}}{2}\left[K(1-K)+\beta_{1} m_{0}\left(m_{0}-K-1\right)\right] \\
& d_{4}=2\left\{\left[\left(\beta_{1}+K-2\right)(K-1)-m_{0} \beta_{1}\right]\left(\xi_{20}+\frac{\xi_{22}}{2}\right)+\left(\beta_{1}+K-2\right) \beta_{20}+\right. \\
& \left.+\frac{\beta_{22}}{2}\left(\beta_{1}+K-1-h_{0}^{\prime}\right)-\frac{3}{4}\left(m_{0} \beta_{1}+\frac{K-1}{2}\right)\left(\beta_{1}+K-1\right)+\frac{K-1}{8}+\frac{3}{8} m_{0}^{2} \beta_{1}\right\} \\
& +\frac{(1-n)(K-1)(1-\mathfrak{C})}{2}\left(\frac{q^{2}}{4}+3 q \xi_{22}+2 q \xi_{20}\right)+\frac{3}{4} \beta(K-1) q^{4} .
\end{aligned}
$$

\section{References}

[1] Mullins, W. W. and Sekerka, R. F., J. Appl. Phys. 35 (1964) 444.

[2] Wollkind, D. J. and Segel, L. A., Philos. Trans. R. Soc. 268 (1970) 351.

[3] Caroli, B., Caroli, C. and Roulet, B., J. Physique 43 (1982) 1767.

[4] Elliott, W. A., Gagliano, F. P. and Krauss, G., Metall. Trans. 4 (1973) 2031.

[5] Boettinger, W. J., Schechtman, D., Shaefer, R. J. and Biancaniello, F. S., Metall. Trans. 15A (1984) 55.

[6] De Cheveigne, S., Guthmann, C. and Lebrun, M. M., to be published in J. Crys. Growth.

[7] Caroli, B., Caroli, C., Misbah, C. and Roulet, B., J. Physique 46 (1985) 1657.

[8] Taylor, N. W., J. Am. Chem. Soc. 45 (1923) 462.

[9] See for example, KuBo, R., Thermodynamics (NorthHolland, Amsterdam) 1968, chap. IV.

[10] HANSEn, M., ANDERKo, K., Constitution of binary alloys (Mc Graw-Hill, New York) 1958, p. 1113.

[11] Hildebrand, J. H. and Sharma, J. N., J. Am. Chem. Soc. 51 (1929) 469.

[12] Tedrow, P. M. and Lee, D. M., in : Proc. LT9 (Part A),
Eds. J. G. Daunt, D. O. Edwards, F. J. Milford, M. Yaqub (Plenum Press, New York, 1965).

[13] Edwards, D. O., Mc Williams, A. S. and DaunT, J. G., Phys. Rev. Lett. 9 (1962) 195 ; Phys. Lett. 1 (1962) 218.

[14] TACONIS, K. W. and DE Bruyn Ouboter, R., in : Prog. Low Temp. Phys. Vol. IV.

[15] Herzlinger, G. A. and King, J. G., Phys. Lett. 40A (1972) 65.

[16] Grilly, E. R., J. Low Temp. Phys. 4 (1971) 615.

[17] Grilly, E. R., J. Low Temp. Phys. 11 (1973) 33.

[18] Gallet, F., Wolf, P. E., Balibar, S., Phys. Rev. Lett. 52 (1984) 2253.

[19] Balibar, S. and Castaing, B., in : Surface Sci. Rep. 5 (1985) 87.

[20] See for example, Prince, A., Alloy Phase Equilibria (Elsevier, Amsterdam) 1966, chap. 3.

[21] Ben-Jacob, E., Goldenfeld, N. D., Langer, J. S. and Schon, G., Phys. Rev. Lett. 51 (1983) 1930 ; Phys. Rev. A 29 (1984) 1335.

[22] Karma, A. and Goldenfeld, N., to be published.

[23] Sivashinsky, G. I., Physica 8D (1983) 243. 